\title{
METHOD OF SPATIAL CHARACTERISTICS IN PROBLEMS OF A MECHANICS OF DEFORMABLE SOLID BODY
}

\author{
Georgy G. Bulychev
}

MIREA-Russian Technological University, https://www.mirea.ru/

Moscow 119454, Russian Federation

geo-bulychev@mail.ru

Abstract. The method of spatial characteristics is considered and analyzed and its possibilities for studing of wave processes in deformable solid bodies of various structure and a rheology are investigated. Merits and demerits of a method are considered, and also the circle of tasks which it is necessary to solve for building of the characteristic form of mathematical model of dynamics and dynamic destruction of researched bodies and for its usage in numerical simulation. The initial and characteristic form of dynamical equations of the anisotropic symmetrically elastic bodies is shown. By means of the considered method numerically on PC the research problem of destruction of the two-storey house is solved at an earthquake load.

Keywords: dynamic processes, a method of spatial characteristics, numerical simulation, dynamics and destruction of buildings and constructions

UDC 539.3

Bibliography - 14 references

Recieved 19.04.2018

RENSIT, 2018, 10(1):77-90

DOI: $10.17725 /$ rensit.2018.10.077

\section{Contents}

1. INTRODUCTION (77)

2. Construction of characteristic forms of DYNAMICS EQUATIONS OF ELASTOVISCO-PLASTIC Bodies. The mathematical apparatus (78)

3. Advantages and disadvantages of the CHARACTERISTIC FORM OF REPRESENTATION OF THE EQUATIONS OF DYNAMICS OF DEFORMABLE SOLIDS (80)

4. Stages of DeVElopment of Computational EXPERIMENT (81)

4.1. Modeling of DyNAMic Destruction of a HOUSE UNDER THE ACTION OF A SEISMIC LOAD (83)

5. Conclusion (88)

REFERENCES (89)

\section{INTRODUCTION}

The method of spatial characteristics (MSC) involves the construction and further use of the characteristic form of the initial equations of dynamics of deformable solids, that is, such a form in which the original equations are converted to the form containing the equations for the waves propagating in the body, and degenerate waves characterizing the non-propagating perturbations (they are called fixed discontinuities). The primary verbal formulation of such a submission was given Ch. Huygens ("Treatise on Light", 1678) and sounded like this: "the Illumination of the body at a point is determined by the sum of the waves coming to this point, and the sources at this point." The mathematical formulation of this statement was obtained 70 years later by D'Alembert as a General solution of the wave equation (formula D'Alembert) and was called the characteristic form, and the method using this form of representation became known as the method of characteristics (for onedimensional dynamic problems) and, later, the method of spatial characteristics (MSC) for multidimensional problems. MSC has been successfully used for solving linear and nonlinear problems of dynamics of gases and liquids. Since the fifties of the 20th century, this method was used for studying the dynamics of deformable solids.

One of the first works in this area is [1], where a matrix apparatus and a diagonalization procedure for symmetric, positive definite matrices were proposed for constructing the characteristic form of a system of hyperbolic equations.

The numerical aspects of the MSC were developed by K.M. Magomedov and A.S. Kholodov for transfertype equations [2]. Based on 
the comparative analysis of different difference schemes, they showed that the dependence of finitedifference characteristic schemes is closest to the dependence of the initial differential equations, that is, the accuracy of calculations using characteristic schemes is higher than using grid. In addition, it was found that, in the first order of approximation (onesided time difference and symmetric coordinate difference), the characteristic finite-difference schemes have a residual member of the parabolic type, which makes them stable when modeling both smooth and discontinuous perturbations. It was also found that the differencecharacteristic schemes of the first order do not create a computational "ripple", resulting in grid schemes in the same cases to oscillatory instability. K. M. Magomedov and A. S. Kholodov school exists and is still developing $[3,4,5,6]$ and with the help of grid-characteristic methods, many practically important problems of gas and hydrodynamics, plasma physics and mechanics of deformed solid state are solved. In the problems of deformed solid mechanics, mainly inverse implicit gridcharacteristic schemes of the first and second order of approximation were used. In the second order of approximation, artificial viscosity was introduced for the purpose of account stability. With the help of such schemes, small and finite deformations of homogeneous, piecewise homogeneous and inhomogeneous bodies, as well as the destruction of such bodies, were considered.

In 1990 the author has constructed the mathematical apparatus, which allows analytically in an explicit form to obtain the characteristic form of equations that anisotropic elastoviscoplastic bodies with symmetrical matrix of rigidity [7]. Thus, the characteristic representation for a large class of deformable solids in the framework of small deformations (the differential part of the initial equations must be linear) was obtained.

Two points turned out to be interesting:

1) the applied apparatus can be used to construct the characteristic form of the equations of dynamics of any medium - gas, liquid, plasma, and solid - if the differential parts of their equations are described by differential invariants of the first order,

2) to solve this problem, it was not necessary either to calculate tensor or to determine the eigenvalues and eigenvectors of matrices, the order of which would exceed the third. Thus, at this stage it was possible to manage with analytical, and identical transformations. With regard to such a (characteristic) form of representation of the initial equations of dynamics, the author developed a numerical method, which is used to solve problems of structural mechanics, one of which is considered as an example in this work.

\section{CONSTRUCTION OF CHARACTERISTIC FORMS OF DYNAMICS EQUATIONS OF ELASTOVISCO-PLASTIC BODIES. THE MATHEMATICAL APPARATUS}

We construct a matrix form of first order's differential invariants, for which we introduce into consideration the scalar $-\varphi$, vector $-\vec{V}$ and symmetric tensor $-\hat{\sigma}$ . We introduce, for simplicity, a Cartesian coordinate system $\left\{x_{i}\right\}$ and present a vector and a tensor as matrix-strings of their physical components:

$$
V=\left\|V_{1}, V_{2}, V_{3}\right\|, \Sigma=\left\|\Sigma_{11}, \Sigma_{22}, \Sigma_{33}, \Sigma_{12}, \Sigma_{13}, \Sigma_{23}\right\|
$$

To select components from these matrix-strings, we use the simplest matrix-strings $e_{i}=\left\|\delta_{1}, \delta_{2 i}, \delta_{3 i}\right\|$, where $\delta_{i j}$ is the unit tensor, and $r_{i j}=\|_{1 i} \delta_{1 i} \delta_{1 j} \delta_{2 i} \delta_{2 p} \delta_{3 i} \delta_{3 p}$ $\delta_{1 i} \delta_{2 j}+\delta_{2 i} \delta_{1}, \delta_{1 i} \delta_{3 j}+\delta_{3 i} \delta_{1}, \delta_{2 i} \delta_{3 j}+\delta_{3 i} \delta_{2 j} \|$, which can be used to select elements from (1): $V_{i}=e_{i} V^{T}, \sum_{i j}=r_{i j} \sum^{T}$, where the superscript " $T$ " indicates transposition.

For the selected objects we will make differential invariants,

$\operatorname{div} \sigma=\left(R_{i} \partial_{j}\right) \Sigma^{T}, \operatorname{def} \vec{V}=\left(\Pi R_{i}^{T} \partial_{i}\right) V^{T}$,

where $\partial_{\mathrm{i}}=\partial / \partial x_{i}$, def $V-$ the operator of deformations, by repeating Roman indices, summation is carried out here and further, $\Pi=$ $\operatorname{diag}(1,1,1,1 / 2,1 / 2,1 / 2)$,

$$
R_{i}=e_{j}^{T} r_{i j}=\left\|\begin{array}{llllll}
\delta_{1 i} & 0 & 0 & \delta_{2 i} & \delta_{3 i} & 0 \\
0 & \delta_{2 i} & 0 & \delta_{1 i} & 0 & \delta_{3 i} \\
0 & 0 & \delta_{3 i} & 0 & \delta_{1 i} & \delta_{2 i}
\end{array}\right\| .
$$

It is easy to notice that the matrices $e_{i}$ and $R_{i}$ have a very simple form, but they determine the topology of wave processes in a symmetrically elastic solid. It is interesting that the transition to the orthogonal curvilinear coordinate system does not increase the number of elementary matrices and also allows the following analytical transformations.

Use equations (2) to construct the characteristic form of the equations of the dynamics of the 
symmetrically-elastic elastoviscoplastic body. The initial system of equations is written in the form of equations of motion, conditions of additivity of deformations, Hooke's law for elastic deformations, Cauchy relations for elastic deformations and the law of plastic flow

$$
\begin{aligned}
& \operatorname{div} \widehat{\sigma}+\vec{f}=\rho \partial_{t} \vec{v}, \widehat{\varepsilon}=\widehat{\varepsilon}^{e}+\widehat{\varepsilon}^{v p}, \widehat{\sigma}=\widehat{c} \cdot \widehat{\varepsilon}^{e}, \\
& \partial_{t} \widehat{\varepsilon}=\operatorname{def} \vec{v}, \partial_{t} \widehat{\varepsilon}^{v p}=\widehat{\Phi}\left(\widehat{\varepsilon}, \widehat{\varepsilon}^{e}, \widehat{\varepsilon}^{v p}, \widehat{\sigma}, \vec{v}, \eta\right) .
\end{aligned}
$$

Here $\vec{f}=\left\|F_{1}, F_{2}, F_{3}\right\|$ - the vector of internal forces, $\rho$ - the density of the body material, $\hat{\sigma}$ - stress tensor, $\vec{V}$ - the velocity vector of the particles of the body, $\widehat{\varepsilon}, \widehat{\varepsilon}^{e}$ and $\widehat{\varepsilon}^{v p}$ - full, elastic and viscoplastic deformation, $\widehat{c}$ - rigidity tensor, $\widehat{\Phi}=\| \Phi_{11}, \Phi_{22}$, $\Phi_{33}, \Phi_{12}, \Phi_{13}, \Phi_{23} \|$ - viscoplastic strain tensor (it is essential that it does not depend on derivatives $\hat{\sigma}$ and $\vec{v}), \partial t=\partial / \partial t$.

It is seen that in the system (3) two differential invariants are divergence (div) and deformation (def), the topology of which is determined by matrices $R$.

With respect to matrix variables $V$ and $\sum$ in the coordinate system $\left\{x_{i}\right\}$, the system (3) can be given in two groups of equations: equations of motion and determining equations

$$
\begin{aligned}
& R_{i} \partial_{i} \Sigma^{T}-\rho \partial_{t} V^{T}=F^{T}, \\
& \partial_{t} \Sigma^{T}=C\left(\Pi R_{i}^{T} \partial_{i} V^{T}-\Phi^{T}\right),
\end{aligned}
$$

where $C$ is the rigidity matrix, $F$ and $\Phi$ matrix-strings of internal forces and velocities of viscoplastic deformations.

Choose the direction $x_{a}$ coinciding with one of the axes of coordinate system $\left\{x_{i}\right\}$ and construct characteristic equations for the waves propagating in both directions along this direction. The remaining axes are called, respectively, $x_{\beta}$ and $x_{\gamma}$; stresses acting on sites with normals corresponding to these axes $\mathrm{R}_{a} \Sigma^{T}, \mathrm{R}_{\beta} \Sigma^{T}, \mathrm{R}_{\gamma} \Sigma^{T}$ are designated, respectively, $\Sigma_{\alpha}^{T}, \Sigma_{\beta}^{T}$ and $\Sigma_{\gamma}^{T}$. Let us denote the matrix $C \Pi$ as $\tilde{C}$ and rewrite (4) as

$$
\begin{aligned}
& \partial_{\alpha} \Sigma_{\alpha}^{T}-\rho \partial_{t} V^{T}=F^{T}-\partial_{\beta} \Sigma_{\beta}^{T}-\partial_{\gamma} \Sigma_{\gamma}^{T}, \\
& \partial_{t} \Sigma^{T}=\tilde{C} R_{i}^{T} \partial_{i} V^{T}-C \Phi^{T} .
\end{aligned}
$$

Multiply the second equation of the system (5) on the left $\tilde{C}_{\alpha \alpha}=R_{\alpha} \tilde{C} R_{\alpha}^{T}, \tilde{C}_{\alpha \beta}=R_{\alpha} \tilde{C} R_{\beta}^{T}, \tilde{C}_{\alpha \gamma}=R_{\alpha} \tilde{C} R_{\gamma}^{T}$ and enter the notation and $R_{\alpha} C \tilde{\Phi}^{T}=\Psi_{\alpha}^{T}$; the second equation (5) is given as

$$
\partial_{t} \Sigma_{\alpha}^{T}-\tilde{C}_{\alpha \alpha} \partial_{\alpha} V^{T}=\tilde{C}_{\alpha \beta} \partial_{\beta} V^{T}+\tilde{C}_{\alpha \gamma} \partial_{\gamma} V^{T}-\Psi_{\alpha}{ }^{T} .
$$

Consider a matrix $\tilde{C}_{\alpha \alpha}$ that is the main minor of the third order of the matrix $\tilde{C}$. The matrix $\tilde{C}$ is always non-degenerate, positively defined and symmetric in construction, hence the matrix $\tilde{C}_{\alpha \alpha}$ has a diagonal decomposition: $\tilde{C}_{\alpha \alpha}=O^{T} \Lambda O$, where $\Lambda=\operatorname{diag}\left(\lambda_{1}, \lambda_{2}, \lambda_{3}\right)$ is the diagonal matrix with positive eigenvalues (let $\lambda_{1}>\lambda_{2} \geq \lambda_{3}$, that is always possible if all elements $\tilde{C}_{\alpha \alpha}$ are positive), and the eigenvector matrices $O$ are orthonormalized and are rotation matrices in the coordinate system $\left\{x_{i}\right\}$.

Let us take $\Lambda=\rho D^{2}$, from where $D= \pm \sqrt{\Lambda / \rho}$ - has the dimension of the velocity and, as will be seen from the further, is a matrix of longitudinal and transverse waves propagating in both directions along the axis $x_{a}$.

Multiply the first equation (5) from left to $|D| O$, equation (6) from left to $O$, add these equations and replace $|D|$ with $\pm D$. After grouping, we obtain the matrix characteristic equations

$$
\begin{aligned}
& \left(\partial_{t} \pm D \partial_{\alpha}\right)\left(O \Sigma_{\alpha}^{T} \mp \rho D O V^{T}\right)= \\
& \partial_{\beta}\left(O \tilde{C}_{\alpha \beta} V^{T} \mp D O \Sigma_{\beta}^{T}\right)+ \\
& +\partial_{\gamma}\left(O \tilde{C}_{\alpha \gamma} V^{T} \mp D O \Sigma_{\gamma}^{T}\right) \pm D O F^{T}-O \Psi_{\alpha}^{T} .
\end{aligned}
$$

Let numerical calculations of equations (7) lead to formulas

$$
O \Sigma_{\alpha}^{T}-\rho D O V^{T}=\Omega_{1}, O \Sigma_{\alpha}^{T}+\rho D O V^{T}=\Omega_{2},(8)
$$

then

$$
\begin{aligned}
& \Sigma_{\alpha}^{T}=O^{T}\left(\Omega_{1}+\Omega_{2}\right) / 2, \\
& V^{T}=O^{T} D^{-1}\left(\Omega_{2}-\Omega_{1}\right) /(2 \rho)
\end{aligned}
$$

and six of the nine defined functions are found (numerically).

To determine the remaining stresses, we introduce a matrix $N_{a}$, obtained by striking out the three rows, corresponding to the matrix $R_{a}$, from the unit matrix sixth order. Then, by construction, $\Sigma_{N}^{T}=N_{\alpha} \Sigma^{T}$ those stresses that remain to be calculated.

Multiply the second equation (5) on the left $N_{a}$ and express $\partial_{a} V^{T}$ from (6); as a result, after grouping, we obtain a matrix degenerate characteristic equation that determines the last three stresses $\Sigma_{N}^{T}$.

$$
\begin{aligned}
& \partial_{t}\left(\Sigma_{N}^{T}-\tilde{C}_{N \alpha} \tilde{C}_{\alpha \alpha}^{-1} \Sigma_{\alpha}^{T}\right)=\left(\tilde{C}_{N \beta}-\tilde{C}_{N \alpha} \tilde{C}_{\alpha \alpha}^{-1} \tilde{C}_{\alpha \beta}\right) \partial_{\beta} V^{T}+ \\
& +\left(\tilde{C}_{N \gamma}-\tilde{C}_{N \alpha} \tilde{C}_{\alpha \alpha}^{-1} \tilde{C}_{\alpha \gamma}\right) \partial_{\gamma} V^{T}+\tilde{C}_{N \alpha} \tilde{C}_{\alpha \alpha}^{-1} \Psi_{\alpha}^{T}-\Psi_{N}^{T} .
\end{aligned}
$$

Here it is marked: 
$\tilde{C}_{N \alpha}=N_{\alpha} \tilde{C} R_{\alpha}^{T}, \tilde{C}_{N \beta}=N_{\alpha} \tilde{C} R_{\beta}^{T}$,

$\tilde{C}_{N \gamma}=N_{\alpha} \tilde{C} R_{\gamma}^{T}, \Psi_{N}^{T}=N_{\alpha} C \tilde{\Phi}^{T}$.

Most of the materials used in industry and construction are isotropic, transversally isotropic or orthotropic. For such materials matrix $\tilde{C}$ has a very simple form

$$
\tilde{C}=\left\|\begin{array}{|cccccc}
c_{11} & c_{12} & c_{13} & 0 & 0 & 0 \\
c_{12} & c_{22} & c_{23} & 0 & 0 & 0 \\
c_{13} & c_{23} & c_{33} & 0 & 0 & 0 \\
0 & 0 & 0 & c_{44} & 0 & 0 \\
0 & 0 & 0 & 0 & c_{55} & 0 \\
0 & 0 & 0 & 0 & 0 & c_{66}
\end{array}\right\|
$$

and $\tilde{C}_{11}=\operatorname{diag}\left(c_{11}, c_{44}, c_{55}\right), \tilde{C}_{22}=\operatorname{diag}\left(c_{44}, c_{22}, c_{66}\right)$, $\tilde{C}_{33}=\operatorname{diag}\left(c_{55}, c_{66}, c_{33}\right)$, and therefore are eigenvectors of the identity matrix of the third order and the equations (7) and (9) splits into 9 scalar characteristic equations

$$
\begin{aligned}
& \left(\partial_{\alpha} \mp c_{1}^{-1} \partial_{t}\right)\left(\Sigma_{\alpha \alpha} \pm \rho c_{1} V_{\alpha}\right)+\partial_{j}\left(\Sigma_{\alpha j} \pm v_{1} \rho c_{1} V_{j}\right)+ \\
& +\partial_{k}\left(\Sigma_{\alpha k} \pm v_{2} \rho c_{1} V_{k}\right)=\tilde{F}_{\alpha} \pm \rho c_{1}\left(\Phi_{\alpha \alpha}+v_{1} \Phi_{j j}+v_{2} \Phi_{k k}\right), \\
& \left(\partial_{\alpha} \mp c_{2}^{-1} \partial_{t}\right)\left(\Sigma_{\alpha j} \pm \rho c_{2} V_{j}\right)+\partial_{j}\left(\Sigma_{j j} \pm \rho c_{2} V_{\alpha}\right)+ \\
& +\partial_{k} \Sigma_{j k}=F_{j} \pm 2 \rho c_{2} \Phi_{\alpha j}, \\
& \left(\partial_{\alpha} \mp c_{3}^{-1} \partial_{t}\right)\left(\Sigma_{\alpha k} \pm \rho c_{3} V_{k}\right)+\partial_{k}\left(\Sigma_{k k} \pm \rho c_{3} V_{k}\right)+ \\
& +\partial_{j} \Sigma_{j k}=F_{k} \pm \rho c_{3} \Phi_{\alpha k}, \\
& \partial_{t}\left(\Sigma_{j j}-v_{1} \Sigma_{\alpha \alpha}\right)=\eta_{1}\left(\partial_{j} V_{j}-\Phi_{j j}\right)+\eta_{2}\left(\partial_{k} V_{k}-\Phi_{k k}\right), \\
& \partial_{t}\left(\Sigma_{k k}-v_{2} \Sigma_{\alpha \alpha}\right)=\eta_{2}\left(\partial_{j} V_{j}-\Phi_{j j}\right)+\eta_{3}\left(\partial_{k} V_{k}-\Phi_{k k}\right), \\
& \partial_{t} \Sigma_{j k}=c_{\chi x}\left(\partial_{j} V_{k}+\partial_{k} V_{j}-\Phi_{j k}\right), \alpha, j, k=1,2,3 ;
\end{aligned}
$$

where $j \neq a, k \neq a, j<k, \beta=a+j+1, \gamma=a+k+1, \chi=j+$ $k+1, c_{1}=\sqrt{c_{\alpha \alpha} / \rho}, c_{2}=\sqrt{c_{\beta \beta} / \rho}, c_{3}=\sqrt{c_{\gamma} / \rho}$, $v_{1}=c_{\alpha j} / c_{\alpha \alpha}, v_{2}=c_{\alpha k} / c_{\alpha \alpha}, \eta_{1}=c_{j j}-v_{1}^{2} c_{\alpha \alpha}$, $\eta_{2}=c_{k j}-v_{1} v_{2} c_{\alpha \alpha}, \quad \eta_{3}=c_{k k}-v_{2}^{2} c_{\alpha \alpha}, \quad F_{i}$ and $\Phi_{i j}-$ physical components of the internal forces vector and similar components of the viscoplastic strain tensor.

The first three pairs of equations (11) describe wave processes on mov-ing discontinuities, that is, for waves propagating in the direction $x_{a}$ of the Cartesian coordinate system $\left\{x_{i}\right\}$ in each pair along the bicharacteristics, two defined variables are changed and, therefore, these variables can be determined independently of the others.
The last three equations of the system (11) independently determine three stresses $\sum_{i,} \sum_{k k}$ and $\sum_{j k}$ through the stress $\Sigma_{a a}$ determined in the first pair of equations. Thus, the characteristic system of equations (11) allows us to de-termine all the stresses and velocities of particles at the inner point of the me-dium under consideration for waves propagating in both directions along the axis $x_{a}$.

\section{ADVANTAGES AND DISADVANTAGES OF THE CHARACTERISTIC FORM OF REPRESENTATION OF THE EQUATIONS OF DYNAMICS OF DEFORMABLE SOLIDS}

Being a generalization of the method of characteristics, MSC has all its advantages:

1. In the characteristic equations, compared with the initial ones, the number of independent variables is reduced by one.

2. Only two variables change along the characteristic: the stress and the particle velocity caused by it, that is, the equations are coupled in pairs for waves propagating along one axis, but in opposite directions.

3. The equations have a divergent form, this allows to use not only grid-characteristic schemes but also integral theorems (Stoks in twodimensional problems and Gauss-Ostrogradsky in three-dimensional problems) and to preserve the conservativeness property in finitedifference schemes in the process of algorithm development.

In terms of numerical implementation, these advantages allow:

1. It is essential to bring together the regions of dependence of the initial differential equations and their finite-difference analogues, thereby sharply increasing the accuracy of calculations (especially in the simulation of wave dynamics and wave destruction).

2. Dramatically reduce the time account and the required amount of RAM.

3. To accurately convey the jumps of the solutions and to track the changing conditions on the discontinuities (cracks, inhomogeneities, boundaries of the media, etc.) until the complete destruction of the element or design.

4. Maintain conservatism of continuous processes. 
5. Do not be afraid of account instabilities when using first order approximation schemes.

Disadvantages of MSC are:

1. A tight connection between the time step and the coordinate step that imposes constraints on the spatial grid. For example, for piecewisehomogeneous media, this grid must also be piecewise homogeneous, with a strictly consistent step.

2. Redundancy of characteristic relations when considering waves in bounded bodies. Thus, for each direction it is possible to construct as many characteristic relations as the defined variables. Therefore, for waves propagating in different directions, an excess number of characteristic equations is obtained. In this case, the choice of the necessary equations is determined either from the context of the problem, or from additional conditions (usually stability and accuracy of computational schemes), or from comparison with known solutions of the same problems.

3. The difficulty of matching the wave propagation area with the curvilinear grid when working in a curvilinear orthogonal coordinate system.

However, the use of characteristic equations (for example, the Gauss-Ostrogradsky formula) to integrate the divergent form largely eliminates this problem

\section{STAGES OF DEVELOPMENT OF COMPUTATIONAL EXPERIMENT}

For the application of MSC to the dynamics simulation and dynamic fracture of solid bodies of complex structure, rheology and geometry required to solve 5 related tasks:

1. Construct a differential characteristic form of the equations of dynamics of the medium.

2. Select the necessary equations and additional conditions in all typical points of this environment.

3. When simulating the fracture dynamics, select the fracture criteria (stratification) and the sequence of conditions on the cracks at different stresses on their banks and the position of the shores themselves.

4. To develop a scheme of project in accordance with paragraphs 2 and 3.
5. To construct characteristic finite-difference schemes in all typical points of the medium and at all possible states of the medium at these points, to make subprograms organizing data input, calculation of the stress-strain state at all points of the spatial grid at successive time points and output of the results in the required form. Combine all this algorithmic material (as a set of subrou-tines) into a single program and test it on problems with known or intuitively known solutions.

To date, the first problem has been solved in the framework of small deformations for all types of symmetric-elastic elastoviscoplastic deformable solids [7]

If waves of several directions propagate in the point under consideration or if any additional conditions are to be fulfilled, the system (11) is overridden and the choice of one or another of the equations included in it can be made only after additional analysis of the processes occurring at this point. Therefore, the method developer encounters the second of these tasks.

For its solution it is necessary to invoke conditions for achieving stability and high precision the characteristic of the computing circuits in typical points of the considered body. Several publications are devoted to these issues [8-10]. So in [8] it was shown that a necessary condition for the stability of the difference characteristic schemes at the interior point of the medium is the use of all independent equations for longitudinal waves propagating at this point. In [9], a characteristic computational scheme at the boundary point was proposed, it was shown that for the construction of this scheme, the equations (11) for which $x_{a}$ coincides with the internal normal to the boundary should be used; the characteristic equations should be supplemented with boundary conditions

$\varsigma_{\delta} \Sigma_{\alpha \delta}+\omega_{\delta} V_{\delta}=\psi_{\delta}, \quad(\delta=1,2,3)$,

which $\varsigma_{\delta}$ and $\omega_{\delta}$ can be constants, functions of coordinates and time or time, and $\psi_{\delta}$ is constant or function.

In [10], in accordance with the instructions [8] and [9], characteristic computational schemes for plane and axisymmetric bodies were constructed at internal and boundary points. It was also shown that in the points on the axis of symmetry the use 
of the Lopital rule to the type uncertainties $V_{R} / R$, $\Sigma_{R Z} / R$ and $\left(\sum_{R R}-\Sigma_{\Theta \Theta}\right) / R$ leads to the fact that some characteristic equations coincide with each other, whereby three of them can be excluded and replaced by conditions $V_{R}=0, \Sigma_{R Z}=0$ and $\Sigma_{R R}=\Sigma_{\Theta \Theta}$.

In [11] for plane and axisymmetric piecewise homogeneous bodies a computational scheme at the point of contact of the layers was proposed. According to this scheme, the computational template at the point of contact of the layers was represented as two schemes of boundary points located in one place in adjacent layers and connected by contact conditions.

$$
\Sigma_{\alpha i}^{+}=\Sigma_{\alpha i}^{-}, V_{i}^{+}=V_{i}^{-}, i=\alpha, \beta \text {. }
$$

Subsequently, this scheme was improved and adapted to the elements of dynamic fracture [12]: it introduced the fracture criteria (separation and shift) and conditions for automatic selection of the sequence of conditions on the contact; it was also generalized to the case of three-dimensional problems. When stratifying depending on the type of fracture, this sequence of conditions is given by means of one of the Tables (1 or 2)

The tables indicate: $\Sigma_{\alpha \alpha}^{+}, \Sigma_{\alpha \alpha}^{-}, \Sigma_{\alpha \beta}^{+}, \Sigma_{\alpha \beta}^{-}-$ normal and tangential stresses on both sides of the fracture extending along the axis $\chi_{\beta} ; u_{\alpha}^{+}, u_{\alpha}^{-}, u_{\beta}^{+}, u_{\beta}^{-}$ displacements along the axes $x_{a}$ and $x_{\beta}$ on both sides of the crack, $\Sigma^{*}, k$ and $\gamma$-experimental constants, $\tau^{*}=-\kappa \Sigma^{*}, \Delta u_{\alpha}=u_{\alpha}^{+}-u_{\alpha}^{-}, \quad V_{\beta}^{+}$and $V_{\beta}^{-}-$the particle velocities along the crack.

Similar to the above, computational schemes were built in some other typical points of bodies, but it should be noted that in General this problem

Table1

Fracture of separation.

\begin{tabular}{|c|c|c|}
\hline \multicolumn{2}{|c|}{ Compression: $\Delta \mathrm{u}_{\alpha}<0$} & Stretching: $\Delta \mathrm{u}_{\alpha} \geq 0$ \\
\hline$\Sigma_{\alpha \alpha}<-\Sigma^{*}$ & $\Sigma_{\alpha \alpha}<-\Sigma^{*}$ & \\
\hline$\Sigma_{\alpha \alpha}^{+}=\Sigma_{\alpha \alpha}^{-}$ & $\Sigma_{\alpha \alpha}^{+}=\Sigma_{\alpha \alpha}^{-}$ & $\Sigma_{\alpha \alpha}^{+}=0$ \\
\hline$\Sigma_{\alpha \beta}^{+}=\tau^{*}$ & $\Sigma_{\alpha \beta}^{+}=\kappa \Sigma_{\alpha \alpha}^{+}$ & $\Sigma_{\alpha \alpha}^{-}=0$ \\
\hline$\Sigma_{\alpha \beta}^{-}=\tau^{*}$ & $\Sigma_{\alpha \beta}^{-}=\kappa \Sigma_{\alpha \alpha}^{-}$ & $\Sigma_{\alpha \beta}^{+}=0$ \\
\hline$V_{\alpha}^{+}=V_{\alpha}^{-}$ & $V_{\alpha}^{+}=V_{\alpha}^{-}$ & $\Sigma_{\alpha \beta}^{-}=0$ \\
\hline
\end{tabular}

is not solved and requires additional analysis of processes at the points under consideration with the involvement of test problems, preferably with an ana-lytical solution, or using other numerical methods.

The problem of selection and joining of spatial grids is related to the fact that in the MSC the time step $h_{t}$ and the coordinate step $x_{a}-b_{a}$ are related by the ratio $b_{a}=c_{a} \cdot b_{p}$, where $c_{a}$ is the speed of longitudinal waves in the direction $x_{a}$.

Therefore, the choice of a single time step for an inhomogeneous or anisotropic body requires different coordinate steps both for different directions (due to anisotropy) and, possibly, for the transition from point to point (due to inho-mogeneity)

When joining spatial grids at the interface of media with different physical and mechanical properties, there is a mismatch of grid nodes on different sides of the boundary, so you have to use the interpolation procedure between the nodes, which leads to a decrease in the accuracy of calculations at the points on the interface.

The rigid connection between the grid steps in time and coordinates is also in-convenient for bodies with curved boundaries, since the coordinate grid is not uniform for such bodies. For such bodies, it is also necessary to use interpola-tion of the calculated grid nodes between the nodes of the coordinate grid, and throughout the body, or use integral theorems.

Let us consider an example of the use of MSC in the simulation of the dynamic destruction of a complex object.

Shear fracture.

\begin{tabular}{|c|c|}
\hline Compression: $\Delta \mathrm{u}_{\alpha}<0$ & Stretching: $\Delta \mathrm{u}_{\alpha} \geq 0$ \\
\hline$\Sigma_{\alpha \alpha}^{+}=\Sigma_{\alpha \alpha}^{-}$ & $\Sigma_{\alpha \alpha}^{+}=0$ \\
\hline$\Sigma_{\alpha \beta}^{+}=-\gamma\left|\Sigma_{\alpha \alpha}^{+}\right| \operatorname{sgn}\left(V_{\beta}^{+}-V_{\beta}^{-}\right)$ & $\Sigma_{\alpha \alpha}^{-}=0$ \\
\hline$\Sigma_{\alpha \beta}^{-}=\gamma\left|\Sigma_{\alpha \alpha}^{-}\right| \operatorname{sgn}\left(V_{\beta}^{+}-V_{\beta}^{-}\right)$ & $\Sigma_{\alpha \beta}^{+}=0$ \\
\hline$V_{\alpha}^{+}=V_{\alpha}^{-}$ & $\Sigma_{\alpha \beta}^{-}=0$ \\
\hline
\end{tabular}

Table 2 


\subsection{Simulation of dynamic destruction of the house under the action of seismic load}

The plan of the simulated two-storey building is shown in Fig. 1 $a, b$, the figures indicate the window door and hatch openings. Window openings in both figures are the same, and the same doorways. Doorways marked with: figures $1-3$ and 9,10; window openings are marked 4-7 and 11-15. Hatch opening indicated by dashed lines and has a numeral 8. The left pic-ture corresponds to the plan of the first floor of the building, and the right - the second. All openings and internal boundaries of structures are assumed to be stress-free. The building is considered as a two-layer elastoviscoplastic material (Foundation and building) with different characteristics of layers. It is assumed that the seismic load acts on the lower surface of the Foundation. The shape of the seismic pulse is a triangle. The rise time is 3 times less than the fall time. The pulse duration is large compared to the time of run on any surface of the house and is equal to the simulation time. In the simulation of the dynamics of the pulse amplitude is much less than the yield strength of the shear materials at home. indicated by dashed lines and has a numeral 8 . The left picture corresponds to the plan of the first floor of the building, and the right - the second. All openings and internal boundaries of structures are as-sumed to be stress-free. The building is considered as a two-layer elastic-viscous plastic material (Foundation and building) with different characteristics of layers. It is assumed

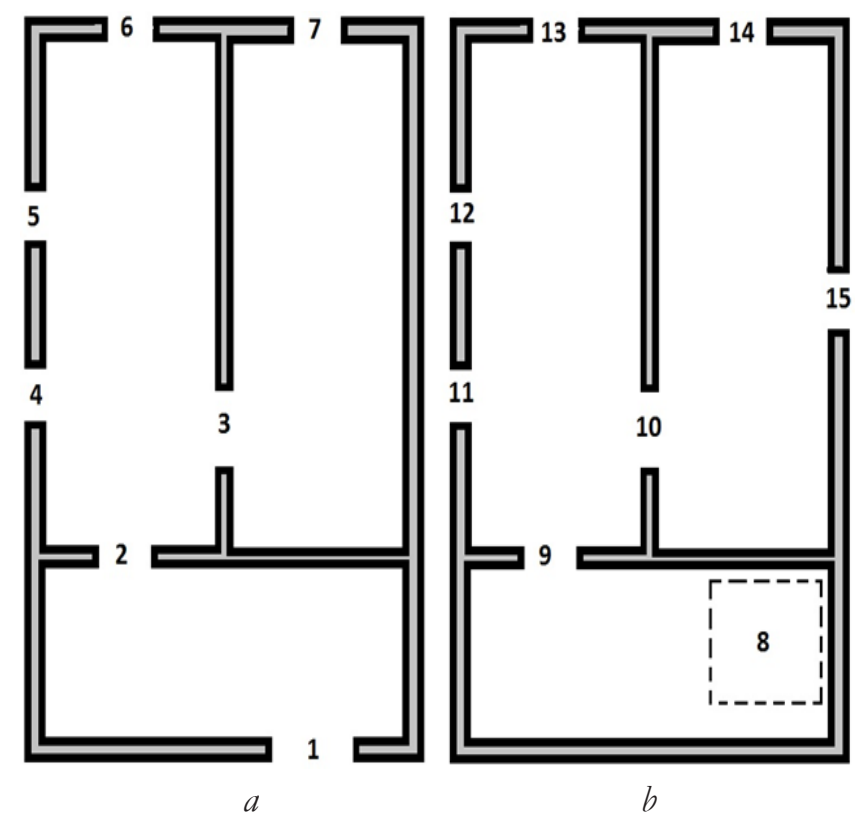

Fig. 1. Ground (a) and second (b) floors of the building. that the seismic load acts on the lower surface of the Foundation. The shape of the seismic pulse is a triangle. The rise time is 3 times less than the fall time. The pulse duration is large compared to the time of run on any surface of the house and is equal to the simulation time. In the simulation of the dynamics of the pulse amplitude is much less than the yield strength on the shear for materials of home

Model and method. The mathematical model of the dynamics of the building material and the foundation consists of the equations of motion, the conditions of the additivity of elastic and viscoplastic deformations, the Cauchy equations, the plasticity law and the Guk law for elastic deformations.

$$
\begin{aligned}
& \partial_{j} \sigma_{i j}^{k}=\rho^{k} \partial_{t} V_{i}^{k}, \varepsilon_{i j}^{k}=\left(\varepsilon_{i j}^{k}\right)^{e}+\left(\varepsilon_{i j}^{k}\right)^{v p}, \\
& \sigma_{i j}^{k}=2 \mu^{k}\left(\varepsilon_{i j}^{k}\right)^{e}+\lambda^{k} \delta_{i j}\left(\varepsilon_{m m}^{k}\right)^{e}, \\
& \partial_{t} \varepsilon_{i j}^{k}=\left(\partial_{j} V_{i}^{k}+\partial_{i} V_{j}^{k}\right) / 2, \\
& \left(\varepsilon_{i j}^{k}\right)^{v p}=\Phi^{k}\left(\sigma_{i j}^{k}\right), \quad i, j=1,2,3, k=1,2,
\end{aligned}
$$

where $\sigma_{i j}^{k}, \varepsilon_{i j}^{k}, V_{i}^{k}, \rho^{k}$-stress, strain in the k-layer, the speed of the particles and the density of the material in the k-layer, $\mu^{k}$ and $\lambda^{k}$ - the parameters of the Lame, $S^{\alpha}=\sqrt{s_{i j}^{\alpha} s_{i j}^{\alpha} / 2}$ - the intensity of stress in the body, $\Phi^{k}\left(\sigma_{i j}^{k}\right)=\Phi^{k}\left(S^{k}-k_{S}^{k}\right)$ - function of plasticity, $s_{i j}^{k}=\sigma_{i j}^{k}-\delta_{i j} p^{k}-$ the stress deviator, $p^{k}=\sigma_{i i}^{k} / 3$ - hydrostatic pressure, $k_{S}^{k}$ static yield strength of the material of the body during the shift.

It is seen that the model (14) in the Cartesian coordinatesystemisreduced to themodelof anisotropic body with the following simplifications in the matrix $\tilde{C}: \quad c_{11}^{k}=c_{22}^{k}=c_{33}^{k}=2 \mu^{k}+\lambda^{k}, c_{44}^{k}=c_{55}^{k}=c_{66}^{k}=\mu^{k}$, $c_{12}^{k}=c_{13}^{k}=c_{23}^{k}=\lambda^{k}, v^{k}$ - Poisson's coefficient, $v_{1}^{k}=v^{k} /\left(1-v^{k}\right), v_{2}^{k}=1-v_{1}^{k}, \tilde{F}_{j}^{k}=0$, $\tilde{\Phi}_{\alpha j}^{k}=\gamma^{k} \sqrt{S^{k}-k_{S}^{k}} \cdot s_{\alpha j}^{k} / S$ and $\tilde{\Phi}_{\alpha j}^{k}=0$ at $S^{k}<k_{S}^{k}$.

Consequently, the characteristic form (14) is reduced to (11) with the above simplifications.

We will carry out the dimensionalization of the characteristic form of the system (14) using the relations 
$\tilde{x}_{i}=x_{i} / x_{0}, \tau^{k}=c_{0}^{k} t / x_{0}, \tilde{\sigma}_{i j}^{k}=\sigma_{i j}^{k} / k_{S}^{k}$,

$\tilde{S}^{k}=S^{k} / k_{S}^{k}, \tilde{V}_{i}^{k}=V_{i}^{k} / V_{S}^{k}, k_{S}^{k}=\rho^{k} c_{0}^{k} V_{S}^{k}$,

$\gamma^{k}=x_{0} /\left(V_{S}^{k} \tau_{0}^{k}\right)$

in which $x_{0}$ - the normalization constant, for which the size of the structure along the axis $x_{1}$ is taken $\left(x_{0}=l\right.$, where $l-$ the maximum linear size of the structure), $V_{S}^{k}$ - the speed of the material particles at which the plastic flow begins $\alpha, i, j=1,2,3, k=1$, 2; for the repeated Greek indices, the summation is not made, $c_{0}^{k}=\sqrt{\left(2 \mu^{k}+\lambda^{k}\right) / \rho^{k}}$ - the longitudinal wave velocity in the layer, $\tau_{0}^{k}-$ the flow delay time.

After these simplifications and obeying, this system will take the form: for a longitudinal wave moving at a speed $c_{0}^{k}$ in both directions along the axis $x_{\alpha}$

$$
\begin{aligned}
& \left(\partial / \partial \tau^{k} \pm \partial / \partial x_{\alpha}\right)\left(\sigma_{\alpha \alpha}^{k} \mp V_{\alpha}^{k}\right)= \\
& =\partial / \partial x_{\beta}\left(v_{1}^{k} V_{\beta}^{k} \pm \sigma_{\alpha \beta}^{k}\right)+\partial / \partial x_{\gamma}\left(v_{1}^{k} V_{\gamma}^{k} \pm \sigma_{\alpha \beta}^{k}\right)- \\
& -\Phi_{\alpha \alpha}^{k}-v_{1}^{k}\left(\Phi_{\beta \beta}^{k}+\Phi_{\gamma \gamma}^{k}\right),
\end{aligned}
$$

for transverse waves propagating at a speed $c_{\perp}^{k}=\sqrt{\mu^{k} / \rho^{k}}$ along the same axis

$$
\begin{aligned}
& \left(\partial / \partial \tau^{k} \pm \xi^{k} \partial / \partial x_{\alpha}\right)\left(\sigma_{\alpha \beta}^{k} \mp \xi^{k} V_{\beta}^{k}\right)= \\
& =\partial / \partial x_{\beta}\left(v_{2}^{k} V_{\alpha}^{k} / 2 \pm \xi^{k} \sigma_{\beta \beta}^{k}\right) \pm \\
& \pm \partial / \partial x_{\gamma}\left(\xi^{k} \sigma_{\beta \gamma}^{k}\right)-\Phi_{\alpha \beta}^{k}, \\
& \left(\partial / \partial \tau^{k} \pm \xi^{k} \partial / \partial x_{\alpha}\right)\left(\sigma_{\alpha \gamma}^{k} \mp \xi^{k} V_{\gamma}^{k}\right)= \\
& =\partial / \partial x_{\gamma}\left(v_{2}^{k} V_{\alpha}^{k} / 2 \pm \xi^{k} \sigma_{\gamma \gamma}^{k}\right) \pm \\
& \pm \partial / \partial x_{\beta}\left(\xi^{k} \sigma_{\beta \gamma}^{k}\right)-\Phi_{\alpha \gamma}^{k},
\end{aligned}
$$

for stationary breaks with normal $x_{a}$

$$
\begin{aligned}
& \partial / \partial \tau^{k}\left(\sigma_{\beta \beta}^{k}-v_{1}^{k} \sigma_{\alpha \alpha}^{k}\right)= \\
& =v_{2}^{k}\left(1-v^{k}\right)^{-1}\left(\partial V_{\beta}^{k} / \partial x_{\beta}+v^{k} \partial V_{\gamma}^{k} / \partial x_{\gamma}-\right. \\
& \left.-\Phi_{\beta \beta}^{k}-v^{k} \Phi_{\gamma}^{k}\right), \\
& \partial / \partial \tau^{k}\left(\sigma_{\gamma \gamma}^{k}-v_{1}^{k} \sigma_{\alpha \alpha}^{k}\right)= \\
& =v_{2}^{k}\left(1-v^{k}\right)^{-1}\left(\partial V_{\gamma}^{k} / \partial x_{\gamma}+v^{k} \partial V_{\beta}^{k} / \partial x_{\beta}-\right. \\
& \left.-\Phi_{\gamma \gamma}^{k}-v^{k} \Phi_{\beta \beta}^{k}\right), \\
& \partial / \partial \tau^{k} \sigma_{\beta \gamma}^{k}= \\
& =v_{2}^{k}\left[\left(\partial V_{\beta}^{k} / \partial x_{\gamma}+v^{k} \partial V_{\gamma}^{k} / \partial x_{\beta}\right) / 2-\Phi_{\beta \gamma}^{k}\right],
\end{aligned}
$$

where $\xi^{k}=c_{\perp}^{k} / c_{0}^{k}$ is the ratio of the transverse wave velocity $c_{\perp}^{k}=\sqrt{\mu^{k} / \rho^{k}}$ to the longitudinal wave velocity $c_{0}^{k}$. The "wave" icon above the functions is removed, because in the future, if not specified, these normalizations will be used.

Note, however, that the formulas (16)-(20) cannot be used directly if (as in this case) the structures have piecewise homogeneous structure. This is due to the fact that the normalization of time in each layer is different and depends on the speed of sound $c_{0}^{k}$ in it. To exclude this dependence, we will perform the following transformations:

1. Choose $c_{0}=\max _{k} c_{0}^{k}$ and divide everything $c_{0}^{k}$ into $c_{0}$, denote $\bar{c}_{0}^{k} \equiv c_{0}^{k} / c_{0}$.

2. Let's see $\tau=c_{0} t / x$, then $\tau^{k}=\bar{c}_{0}^{k} \tau$.

3. Multiply all equations (16) - (20) by $\bar{c}_{0}^{k}$ and denote $x_{\eta}^{k}=x_{\eta} / \bar{c}_{0}^{k}, \quad \eta=\alpha, \beta, \gamma$. Denote $\bar{\gamma}^{\alpha} \equiv \bar{c}_{o}^{\alpha} \gamma^{\alpha}$.

Consider the resulting equations using the introduced relations. For example, the equation (16) in the new notation will take the form

$$
\left(\partial / \partial \tau \pm \partial / \partial x_{\alpha}^{k}\right)\left(\sigma_{\alpha \alpha}^{k} \mp V_{\alpha}^{k}\right)=
$$

$$
\begin{aligned}
& =\partial / \partial x_{\beta}^{k}\left(v_{1}^{k} V_{\beta}^{k} \pm \sigma_{\alpha \beta}^{k}\right)+\partial / \partial x_{\gamma}^{k}\left(v_{1}^{k} V_{\gamma}^{k} \pm \sigma_{\alpha \beta}^{k}\right)- \\
& -\bar{F}_{\alpha \alpha}^{k}-v_{1}^{k}\left(\bar{F}_{\beta \beta}^{k}+\bar{F}_{\gamma \gamma}^{k}\right),
\end{aligned}
$$

where $\bar{\Phi}_{\eta \varsigma}^{k}=\bar{\gamma}^{k} \frac{\sqrt{S^{k}-1}}{S^{k}} s_{\eta \varsigma}^{k}, \quad \eta, \varsigma=\alpha, \beta, \gamma$.

The rest of the equations will change in the same way.

Let's set the time change $\Delta \tau$, then the position of the wave front in the $k$-th layer will change by the value $\Delta x^{\alpha}= \pm \Delta \tau \cdot \bar{c}_{0}^{\alpha}$. This relationship determines the relationship between time and space grids used in numerical simulation of dynamics and dynamic fracture of piecewise homogeneous isotropic bodies by the method of spatial characteristics. Observe, that all values under the differential sign do not change, and algebraic terms are simply multiplied by a constant $\bar{c}_{0}^{\alpha}$; by virtue of the isotropy of the body, this constant is the same for all directions of wave propagation.

The given reasoning shows that the algorithms developed for homogeneous isotropic bodies can be used in the internal points of piecewise homogeneous bodies, only the spatial grid and the internal points of the some constants that are specified as input. 
At the points of the interface of the layers, the computational schemes can be obtained by upgrading the schemes describing the occurrence and propagation of cracks along this interface. At the same time on the interface between $k$-th and $(k+1)$-th layers with the unit vector of the normal $n$ and tangent $\tau$, depending on the stress-strain state in its vicinity and the presence or absence of cracks on it, the conditions of continuity are fulfilled:

$$
\bar{V}_{i}^{k}=a \bar{V}_{i}^{k+1}, \bar{\Sigma}_{i n}^{k}=b \bar{\Sigma}_{i n}^{k+1},
$$

where

$$
a=V_{S}^{k+1} / V_{S}^{k}, b=k_{S}^{k+1} / k_{S}^{k},
$$

or the conditions of the free surface

$$
\bar{\sigma}_{i n}^{k}=\bar{\sigma}_{i n}^{k+1}=0 \text {, }
$$

or conditions of Coulomb friction:

$$
\begin{aligned}
& \bar{V}_{n}^{k}=a \bar{V}_{n}^{k+1}, \bar{\Sigma}_{n n}^{k}=b \bar{\Sigma}_{n n}^{k+1}, \bar{\Sigma}_{n \tau}^{k}=-\bar{\Sigma}_{n \tau}^{k+1}, \\
& \bar{\Sigma}_{n \tau}^{k+1}=\omega^{k} \cdot\left|\bar{\Sigma}_{n n}^{k+1}\right| \operatorname{sgn}\left(V_{\tau}^{k+1}-V_{\tau}^{k}\right),
\end{aligned}
$$

where $\tau \neq n$ and $\omega^{\mathrm{k}}<1$ is the dry friction coefficient.

It is easy to see that the ratios (22) to (24) are a generalization of the ratios given in Tables 1 and 2 .

Another important problem that arises when modeling dynamics at the interface of layers is the mismatch of grid nodes on different sides of the interface. This problem is solved with the help of linear interpolation schemes of control points: first, the reference points of $k+1$ layer are interpolated so as to obtain a calculation scheme for the contact surface from the $k$-that layer and with their help are calculated $\sum_{i j}$ and $V_{i}$ on the $k$-that layer (these values are used in the future for calculations on the $k$-that layer), then interpolated reference points on the $k$-that layer and with their help are calculated $\Sigma_{\mathrm{ij}}$ and $V_{i}$ on the $k+1$ layer and they are used for calculations on the $k+1$ layer. In the calculation schemes themselves the stress and displacement analysis at the internal contact boundaries is carried out and on the basis of this analysis a decision is made to apply one or another of the relations (22)-(24).

The criteria for the formation of cracks in the internal points of the structure are selected taking into account the accumulation of damage in the form $J_{n}=A$ or $J_{\tau}=B$, where $\left.J_{n}=\int_{\tau_{0}}^{\tau}<\sigma_{n}(\tau) / \sqrt{3}-1\right\rangle d \tau$, $J_{\tau i}=\int^{\tau}<\sigma_{\tau i}(\tau)-1>d \tau, \quad i=1,2$, constants $A$ and

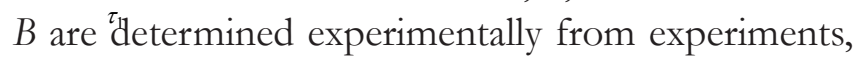

and the direction of the normal $n$ or tangents $\tau$ is determined by the results of the simulation of the dynamics.

Here $\langle f\rangle=f$ at $f>0$ and $\langle f\rangle=0$ at $f \leq 0 ; A=B$ $=1 ; \tau_{0}$ and $\tau_{1}$ are the moments of time in which the appropriate tension for the first time in the plastic region.

Until the failure criteria are met, the continuity of particle velocities and stress-es is assumed at all internal points of the constructions' and foundations. It is further assumed that the crack develops according to the conditions (22)-(24) in which $a=b=1$.

Boundary and initial conditions. The load consists of two parts: the actual seismic wave $P_{\lambda}(t)$ shown in Fig. 5 the dashed line, and the elastic response of the soil to the foundation, expressed by the Winkler formula, in which, due to the specifics of the adopted form of the equations of the model, the ratio between the normal stress on the boundary of the foundationsoil and the normal movement of the soil $-\rho^{1}\left(c_{0}^{1}\right)^{2}$ is normalized to $k$, where the index unit corresponds to the foundation. The weight of the structure and foundation is not taken into account, as it is believed that it is balanced by the static reaction of the soil and does not change in the process of modeling.

The General formula of loading at each point $\left\{x_{2}, x_{3}\right\}$ of the foundation $\left(x_{1}=0\right)$ has the form

$\mathrm{P}\left(t, x_{2}, x_{3}\right)=k u^{1}\left(t, x_{2}, x_{3}\right)-P(t) \leq 0$ and $P\left(t, x_{2}, x_{3}\right)=0$ (25) at $k \cdot u\left(t, x_{2}, x_{3}\right)-P_{(}(t)>0$, where $u^{1}\left(t, 0, x_{2}, x_{3}\right)=\int_{0}^{t} V\left(t, 0, x_{2}, x_{3}\right) d t$ the displacement of the bottom point of the foundation down along the axis $x_{1}$. The first formula in (25) refers to such a load, in which the contact between the ground and the base of the structure, the second - when such contact is broken and between the sole and the ground cavity was formed. At the initial moment the building is at rest.

The General scheme of the project is shown in Fig. 2. With its help, two modeling problems are solved:

1. Study of the dynamics of the building under the influence of seismic load.

2. Investigation of its dynamic failure under the same type of load. 


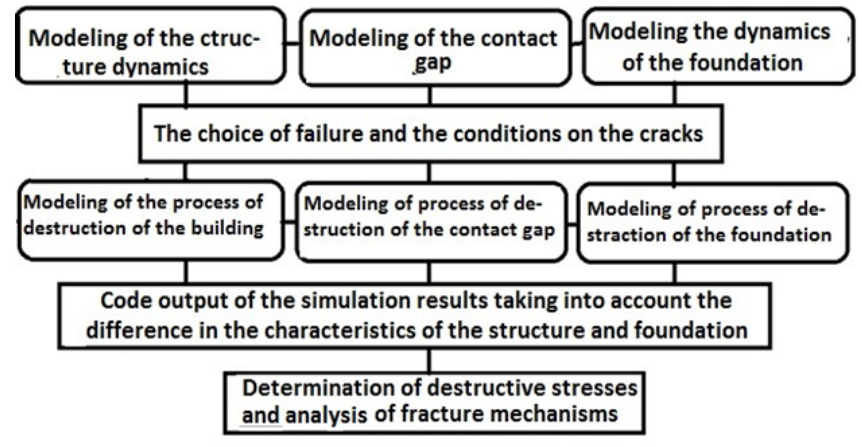

Fig. 2. Block diagram of modeling the dynamic destruction of a structure.

The solution of these tasks is divided into six successive stages.

At the first stage, the dynamics of structures and foundations is being modeled, and their destruction is not envisaged. The load is the normal compressive stresses acting on the foundation and modeling the seismic load [13]. The shape of the loading pulse has the form of stresses $P_{f}=P_{0} \cdot f(t)$, where $f(t)$-a triangular pulse, the amplitude of which is equal to one, the duration corresponds to 30 times of the wave path on the maximum size of the building (which is equal to the simulation time), the amplitude $P_{0}$ has such a value that the destruction, even local, does not occur. The data sets obtained as a result of modeling at this stage are used for graphical processing to determine the maximum stresses and their localization areas.

At the second stage, the specified treatment is carried out. This uses OpenGL primitives and authored contour building routines developed on the basis of the GRAFOR package.

At the third stage, the criteria for the beginning of the fracture and the conditions on the cracks are selected. As criteria for the beginning of failure conditions of accumulation of inelastic normal or tangential stresses to a predetermined value are used. The further behavior of the crack is determined by one of the tables above.

At the fourth stage, the simulation of dynamic structural failure at different amplitudes of loading stresses is carried out $P_{0}$. As a criterion for the complete destruction of the structure is taken to complete the destruction of any of its cross-section. Destruction analysis is performed automatically, and the specified criterion is used to end the account. It is also assumed that the destruction occurs during a time not exceeding 30 times of the longitudinal wave run at the maximum size of the structure.

At the fifth stage, the arrays obtained at the time of complete destruction of the structure are analyzed to determine the areas of catastrophic destruction and the nature of the destruction in them.

And finally, at the sixth, last, stage, the problem of determining the min-imum loads leading to complete destruction of structures is solved.

Modeling parameters. Due to the choice of normalization, variable phys-ical and mechanical parameters are Poisson's ratios $\nu^{1}$ and $\nu^{2}$, accordingly, the foundation and structure, the relative longitudinal velocities $\bar{c}_{0}^{1}$ and $\bar{c}_{0}^{2}$, the relative yield limit for the shift $b$, the relative velocity limit of the beginning of the flow $\alpha$, the coefficients that determine the beginning of the flow $\gamma^{1}$ and $\gamma^{2}$ the dry friction coefficients $-d^{n}$ and $d^{2}$ the time step $-b$. The value $b$ is determined from the normalization of the maximum size of the structure, which is taken as 1 , and the maximum speed, which is also taken as 1. Other parameters are geometrical and determine (in cells) dimensions and spatial structure of buildings and foundations; the size of cells is determined by the spatial grid in the corresponding layers.

The building has cell sizes $(x \times y \times z) 80 \times 144 \times 80$, the thickness of the foundation of 4 cells, and the thickness of the side and upper walls varies and is 2 , 3 or 4 cells, the size of the cell windows $10 \times 10$, the door-cells $20 \times 10$. The dimensions of the cell hatch $24 \times 32$. The arrangement of windows, doors and hatch is shown in the plans Fig. $1 a, b$. The maximum size of the structure is taken to be equal to one.

In the first phase of the simulation, as indicated above, the possibility of destruction was not considered. In different order of layers depending on the thickness of the foundation or the walls of the building, the stresses at the midpoint of the foundation were determined during the whole modeling time.

For Fig. 3-6 shows the evolution of normal stress $\sigma_{11}$ at the midpoint of the base of the structure, $P=-0.01 k$, which corresponds to the compression. Time $\tau$ is measured in the longitudinal waves by the largest size of the structure (here $y$ ). Dotted line in Fig. 5 shows the seismic load. The coefficient $k$ according to the results [14] is chosen to be 0.0005 . 
Figures 3 and 4 correspond to the case when the Foundation is made of a more rigid material

$c_{0}^{1}=1, c_{0}^{2}=0.5, v^{1}=0.1, v^{2}=0.2, a=1, b=0.5$,

$\omega^{1}=0.3, \omega^{2}=0.3, \bar{\gamma}^{1}=0.2, \bar{\gamma}^{2}=0.1$.

The

argument of the graphs is dimensionless time $\tau$, determined by the value - normal voltage $\sigma_{11}$, acting in the same direction as the load. This voltage is normalized to the layer where the applied load.

The parameter to be changed is shown in Fig. 3 is the thickness of the building, which is, respectively, 2, 3, 4 and 5 cells, a fixed parameter is the thickness of the Foundation, which is 3 cells. For Fig. 4 variable parameters is the thickness of the foundation, which varies from 1 to 5 cells, a fixed parameter is the thickness of the walls of the building, which is 3 cells.

For Fig. $\mathbf{5}$ and Fig. $\mathbf{6}$ shows the same stresses $\sigma_{11}$ at the same point of the same structure and at the same load, but in the reverse sequence of materials: here

$$
\begin{aligned}
& c_{0}^{1}=0.5, c_{0}^{2}=1, v^{1}=0.2, v^{2}=0.1, \\
& \omega^{1}=0.3, \omega^{2}=0.3, \bar{\gamma}^{1}=0.1, \bar{\gamma}^{2}=0.2, a=1, b=2 .
\end{aligned}
$$

Fig. 5, as well as Fig. 3 corresponds to the case when the variable parameter is the wall thickness of the building (from 2 to 5 cells), and the invariable - the thickness of the foundation ( 3 cells). For Fig. 6 the variable parameter is the thickness of the foundation: the number of foundation cells in its thickness varies from 1 to 10 , taking into account that the size of the mesh cell in the foundation in this case is 2 times less than in the case shown in Fig. 3; the thickness of the building remains unchanged and is 3 cells.

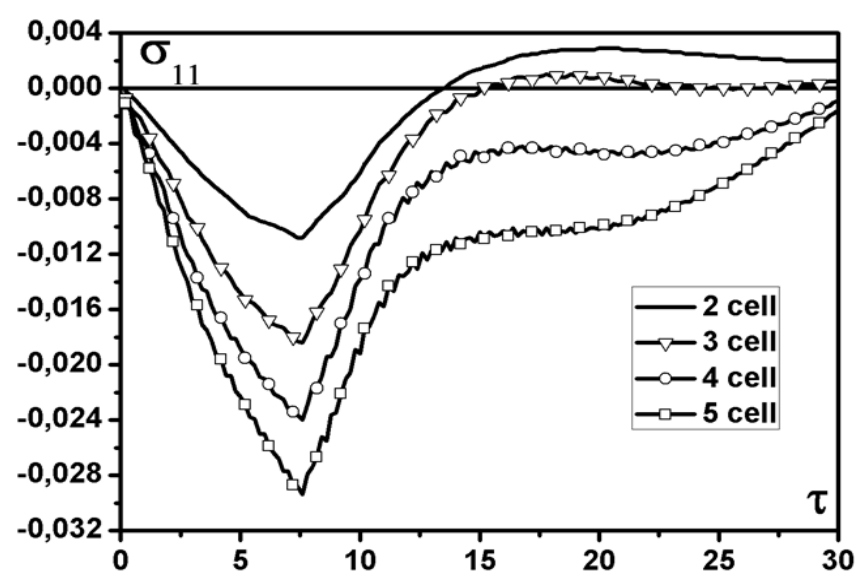

Fig. 3. Hard foundation. The thickness of the walls of the building changes.

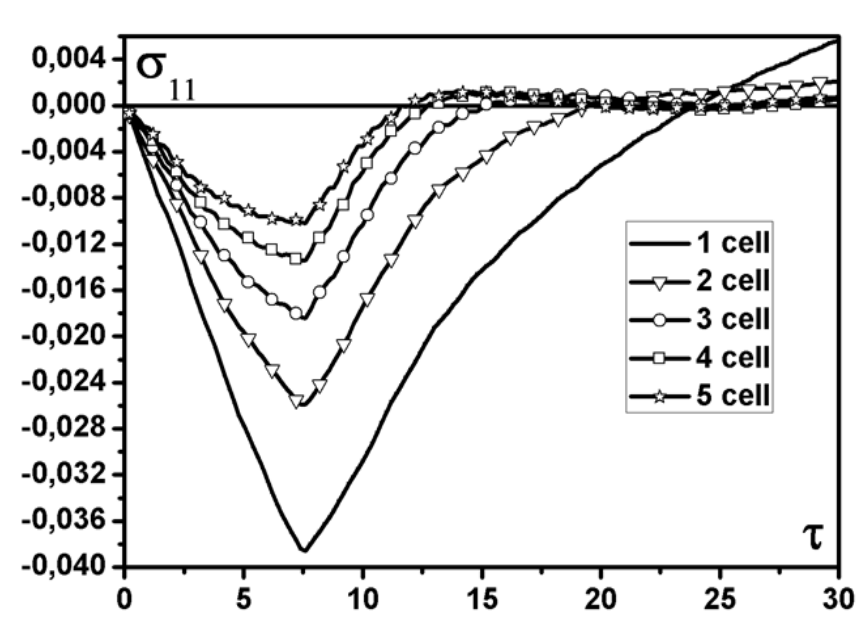

Fig. 4. A hard foundation. The thickness of the foundation varies.

Analysis of the graphs shown in Fig. 3-6 shows that if the Foundation material is stronger than the structure material, the stresses $\sigma_{11}$, generated by the load at the base of the building, are twice less.

In the second statement, the amplitudes of the minimum seismic loads leading to the destruction of structures are determined. When modeling a structure, it is considered to be destroyed if one of its cross sections is completely destroyed. This requirement is sufficient for destruction, but is not necessary. Therefore, the resulting load values are too high. Since the destruction can be complex, 9 fracture criteria are considered: 3 for normal stresses and 6 for tangents. The analysis of fracture mechanisms shows that it is possible both the intersection of cracks (with a partial thermal healing of the material in the intersection region) and the fusion of cracks of the same direction with the formation of a main crack.

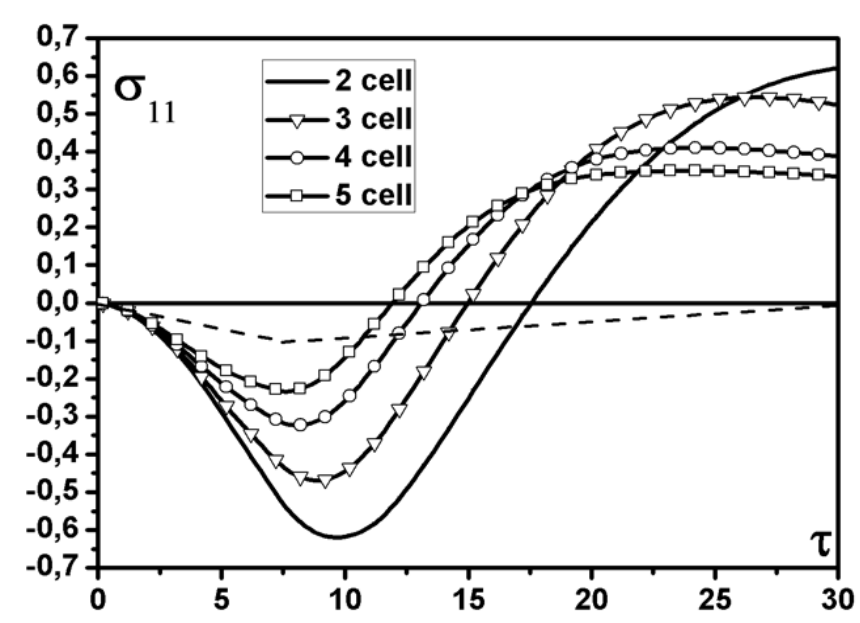

Fig. 5. Soft foundation. The thickness of the walls of the building changes. 


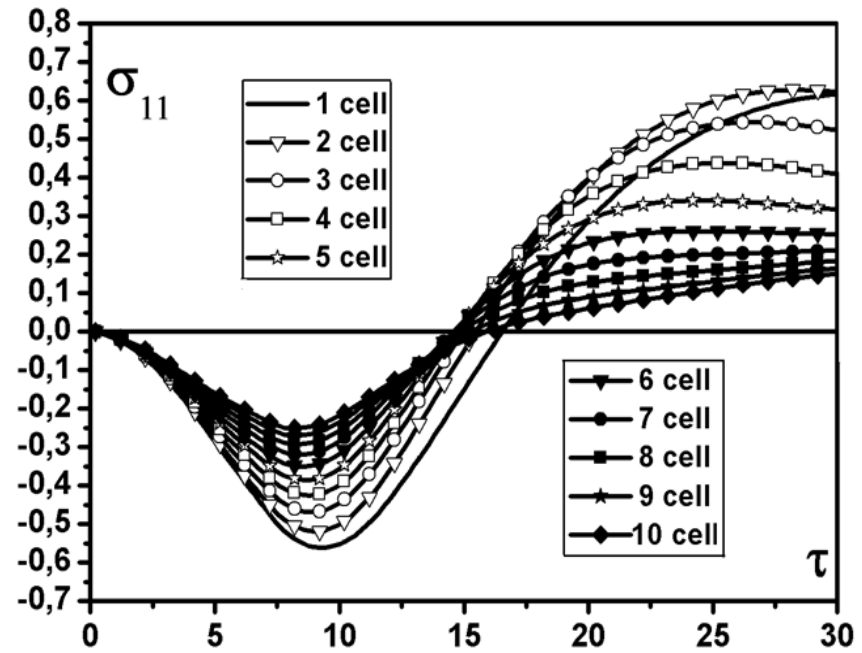

Fig. 6. Soft foundation. The thickness of the foundation is changing.

In the simulation, both the thickness of the Foundation and the thickness of all the walls of the structure were changed. According to the analysis Fig. 3-Fig. 6 it was assumed that the rigidity of the Foundation is higher than the rigidity of the structure $(b=0.5)$.

The following Table 3 shows the values of the minimum amplitudes of seismic loads $P$, leading to complete destruction of the structure. The load $P_{f}$ is shown as a dotted line in Fig. 4. Figures 1-6 vertically indicated, expressed in the number of cells, the thickness of the foundation; Figures 2-6 horizontally indicated the thickness of the walls and walls of buildings (they are the same), also expressed in the cells. The minus sign corresponds to compression.

Analysis of the data in Table 3 shows that at a minimum wall thickness of 2 cells, regardless of the thickness of the foundation, the destructive stresses are extremely small. Further, with the increase in the thickness of the walls, these stresses increase rapidly; the dependence on the thickness of the foundation at the same wall thickness is weaker. The

Table. 3. Minimum loads leading to complete destruction of the structure

\begin{tabular}{|l|l|l|l|l|l|}
\hline Foundation & 2 & 3 & 4 & 5 & 6 \\
\hline 1 & -0.01 & -0.35 & -0.41 & -0.45 & -0.41 \\
\hline 2 & -0.02 & -0.38 & -0.52 & -0.46 & -0.46 \\
\hline 3 & -0.02 & -0.37 & -0.54 & -0.55 & -0.45 \\
\hline 4 & -0.02 & -0.35 & -0.56 & -0.56 & -0.45 \\
\hline 5 & -0.02 & -0.34 & -0.54 & -0.54 & -0.41 \\
\hline 6 & -0.02 & -0.33 & -0.52 & -0.54 & -0.42 \\
\hline
\end{tabular}

variety of possible mechanisms of fracture and the movement of cracks in different directions can lead to their partial healing - when the cracks of different directions intersect, stop each other, or accelerate the destruction, when the cracks of one direction, moving in a straight line, merge. These effects occur when the thickness of the walls or Foundation is large enough - and the minimum destructive stresses $P_{f}$ can fluctuate within significant limits. The maximum breaking load is -0.56 and is achieved with a wall thickness of 4 or 5 cells and a Foundation thickness of 4 cells, with large wall thicknesses due to the merger of cracks of one direction in the Foundation, the minimum breaking loads fall somewhat.

\section{CONCLUSION}

In conclusion, we emphasize once again the properties of the characteristic form of the equations of dynamics of deformable solids, which make it attractive for numerical study:

1. The number of independent variables is reduced by one; the number of calculations and the amount of memory required is drastically reduced. In addition, the areas of dependence of differential equations and their finite-difference analogues are maximally pull together, which allows to increase the accuracy of calculations in the simulation of dynamics. Aptly conveyed by the jump per-turbations. In the first order of approximation, computational schemes are divergent, have positive approximation viscosity, stable and conservative.

2. Block type of differential equations; their differential part is written in the accompanying Cartesian coordinate system, the right part consists of separate blocks: a block of mass forces, a block associated with rheology and a block of geometric terms (in a curvilinear coordinate system). Such a structure is computationally tractable equations allows us to write programs consisting of separate functional modules, and easy tunable, when modifying rheology, mass forces, and the curvature of the coordinate system.

3. The collapse of the characteristic equations for a pair of independent and separate of the equation. For isotropic, transversally isotropic, and orthotropic media, the system of characteristic equations 
decays into a series of independent scalar pairs of equations for the corresponding pairs of unknown stresses and velocities of the particles. These pairs of equations are equations on moving discontinuities, and variables (for waves propagating in the direction $\left.x_{a}\right)-\sum_{a j}$ and $V_{p} j$ $=1,2,3$. Thus further simplifies the form of equations and reduces the volume of calculations, and for the boundary points and points at which the destruction is possible, it is possible to enter into the computational schemes additional conditions - boundary conditions or conditions on the crack, which are determined by the same variables. The remaining equations are separate equations for determining stresses $\sum_{j p}, \sum_{k k}$ and $\sum_{j k}$, moreover, only one of these stresses is included in each equation.

4. In the study of the processes of destruction or stratification, the characteristic form of representation of the initial equations allows the use of a double spatial grid and simulation under different conditions on the boundaries between the cells, which change during the counting process. Thus, not only the formation and movement of the crack can be simulated, but also the multiple destruction of bodies.

The example of modeling the destruction of a two-storey house demonstrates the possibility of using MSC to solve complex important tasks on the PC.

\section{REFERENCES}

1. R. Courant. Partial differential equations. NYLondon, 1962.

2. Magomedov KM, Kholodov AS. Setochnokharakteristicheskie chislennye metody [Gridcharacteristic numerical methods]. Moscow, Nauka Publ., 1988, 288 p.

3. Magomedov KM, Kholodov AS. Setochnokharakteristicheskie chislennye metody: uchebnik. [Gridcharacteristic numerical methods: textbook]. Moscow, Yurayt Publ., 2018, 287 p.

4. Petrov IB, Favorsky AV, Khokhlov NI, Miryakha VA, Sannikov AV, Beklemisheva KA, Golubev VI. Setochno-kharakteristicheskiy metod dlya chislennogo modelirovaniya volnovykh protsessov $\mathrm{v}$ trekhmernykh zadachakh dinamicheskogo nagruzheniya slozhnykh konstruktsiy [Grid-characteristic method for numerical simulation of wave processes in threedimensional problems of dynamic loading of complex structures]. Radioelektronika. Nanosistemy. Informatsionnye tekhnologii (RENSIT), 2015, 7(1):34-47; DOI: 10.17725/ rensit.2015.07.034 (in Russ.).

5. Ivanov AM, Khokhlov NI, Petrov IB. Primenenie tekhnologiyparallelnogo programmirovaniya dlya modelirovaniya seysmicheskikh voln setochnokharakteristicheskim metodom [Application of parallel programming technologies for modeling seismic waves by grid-characteristic method] RENSIT, 2016, 8(2):185-195; DOI: 10.17725/ rensit.2016.08.185 (in Russ.).

6. Egian VS, Favorskaya AV, Mkrtchyan AA, Petrov IB, Khokhlov NI. Golubev VI. Chislennoe modelirovanie processa obnaruzheniya karstovykh polostey $\mathrm{v}$ zheleznodorozhnykh nasypyakh setochno-kharakteristicheskim metodom [Numerical simulation of the process of detection of karst cavities in railway embankments by the grid-characteristic method]. RENSIT, 2017, 9(2):215-220; DOI: 10.17725/ rensit.2017.09.215 (in Russ.).

7. Bulychev GG. Postroenie matrichnoy kharakteristicheskoy formy uravneniy dinamiki anizotropnykh uprugovyazkoplasticheskikh sred [Construction of the matrix characteristic form of the equations of dynamics of anisotropic elastic-viscous-plastic media]. Izvestiya $\mathrm{R} A N$, ser. Mekbanika tverdogo tela. 1995, 1:91-95 (in Russ.).

8. Courant R, Friedrichs K, Lewy H. Über die partiellen Differenzengleichungen der mathematischen Physik. Mathematische Annalen, 1928, 100(1):32-74.

9. Butler DS. The numerical solution of hyperbolic systems of partial differential equations in three independent variables. Proc. Roy. Soc. A, 1960, 255(1281):319-343.

10. Kukudzhanov VN. Chislennoe reshenie neodnomernykh zadach rasprostraneniya voln napryazheniy $\mathrm{v}$ tverdykh telakh [Numerical solution of non-dimensional problems of propagation of stress waves in solids]. Soobshcheniya po prikladnoy matematike VTs $A N$, 1976, 6:67 (in Russ.). 
11. Bulychev GG. Chislennoe issledovanie dinamiki i dinamicheskoy prochnosti kusochnoodnorodnykh uprugoplasticheskikh tel Numerical study of dynamics and dynamic strength of the piecewise-homogeneous elastoplastic bodies]. Stroitelnaya mekhanika $i$ raschet sooruðheniy, 1987, 5:56-59 (in Russ.).

12. BulychevGG, Kukudzhanov VN.Dinamicheskoe razrushenie predvaritelno napryazhennogo voloknistogo kompozita, vyzvannoe obryvom volokna [Dynamic fracture of a prestressed fibrous composite caused by fiber breakage]. Izvestiya RAN, ser. MTT, 1993, 3:207-214 (in Russ.).

13. Kotlyarevsky VA. Raschet nadezhnosti i seysmostoykosti sooruzheniy [The calculation of the reliability and seismic stability of structures]. Stroitelnaya mekhanika $i$ raschet sooruzheniy, 2014, 2:40-46 (in Russ.).

14. Tyapin AG. Raschet dinamicheskogo otryva fundamentnoy plity osnovaniya pri seysmicheskom vozdeystvii. Chast' 2: prosteyshee sochetanie otryva c zapazdyvayushchimi zhestokostyami osnovaniya [The calculation of the dynamic separation of the Foundation plate under seismic action. Part 2: the simplest combination of separation with delayed rigidity of the base]. Stroitelnaya mekbanika $i$ raschet soorugheniy, 2013, 3:39-43 (in Russ.). 